\title{
Correction: UCN-01 induces $S$ and G2/M cell cycle arrest through the p53/p21waf1 or CHK2/CDC25C pathways and can suppress invasion in human hepatoma cell lines
}

\author{
Guoyi $\mathrm{Wu}^{1+}$, Linan $\mathrm{Xu}^{2+}, \mathrm{Nan}^{\mathrm{Lin}}{ }^{3^{*}}$, Bo Liu ${ }^{1 *}$ and Mark A Feitelson ${ }^{4}$
}

\section{Correction}

After publication of this work [1], we noted that we inadvertently failed to include the complete list of all co-authors. In addition, it has been brought to our attention that an Authors Contributions section was not included in the published article. In this correction we rectify these mistakes. The full list of authors and the Authors Contributions section are reported below and the Acknowledgements section has been modified accordingly. We apologize for any inconvenience this oversight may have caused.

\section{Corrected Authors' List:}

Guoyi $\mathrm{Wu}(1)$, Nan Lin(2), Linan $\mathrm{Xu}(3)$, Bo Liu(1) and Mark A. Feitelson(4)

1 Department of General Surgery, the lingnan Hospital, the Third Affiliated Hospital, Sun Yat-Sen University, GuangZhou 510630, PR China

2 Department of Hepatobiliary Surgery, the Third Affiliated Hospital, Sun Yat-Sen University, GuangZhou, 510630, PR China

3 Department of Gynecology and Obstetrics, the First Affiliated Hospital, Sun Yat-Sen University, GuangZhou 510089, PR China

4 Department of Biology, College of Science and Technology, Temple University, Philadelphia, PA 19122 USA

\section{Authors' contributions}

GW performed the cell culture and cell cycle analyses, NL participated in the cell cycle analysis and drafted the manuscript. LX performed the immunoassays.

$\mathrm{BL}$ conceived of the study, participated in its design and coordination and helped draft the manuscript. MF conceptualized the study, generated experimental designs for the work, and helped with data interpretation. GW and LX contributed equally to this work. All authors read and approved the final manuscript.

\section{Corrected Acknowledgements:}

This research is supported by National Science Foundation of China (81272642), the National Science Foundation for Young Scholars of China (81000674), Fundamental research funds for the central universities (10120110040) and Natural Science Foundation of Guangdong Province, China (S2011010004729). This work was also supported by the NIH grants Al076535 and CA104025 to MF.

\section{Author details}

'Department of General Surgery, The lingnan Hospital, The Third Affiliated, Hospital, Sun Yat-Sen University, GuangZhou 510630, PR China. ${ }^{2}$ Department of Hepatobiliary Surgery, The Third Affiliated Hospital, Sun Yat-Sen University, GuangZhou 510630, PR China. ${ }^{3}$ Department of Gynecology and Obstetrics, The First Affiliated Hospital, Sun Yat-Sen University, GuangZhou 510089, PR China. ${ }^{4}$ Department of Biology, College of Science and Technology, Temple University, Philadelphia, PA 19122, USA.

Received: 14 March 2014 Accepted: 18 March 2014

Published: 4 April 2014

\section{References}

1. Guoyi W, Linan X, Nan L, Bo L: UCN-01 induces $S$ and G2/M cell cycle arrest through the p53/p21waf1 or CHK2/CDC25C pathways and can suppress invasion in human hepatoma cell lines. BMC Cancer 2013, 13:167.

\section{doi:10.1186/1471-2407-14-216}

Cite this article as: Wu et al:: Correction: UCN-01 induces $S$ and G2/M cell cycle arrest through the p53/p21 waf1 or CHK2/CDC25C pathways and can suppress invasion in human hepatoma cell lines. BMC Cancer 2014 14:216.

\footnotetext{
* Correspondence: linnancn@gmail.com; jakeliubo@gmai.com

${ }^{\dagger}$ Equal contributors

${ }^{3}$ Department of Gynecology and Obstetrics, The First Affiliated Hospital, Sun Yat-Sen University, GuangZhou 510089, PR China

'Department of General Surgery, The lingnan Hospital, The Third Affiliated, Hospital, Sun Yat-Sen University, GuangZhou 510630, PR China
} 